\title{
Early Universe Quantum Processes in BEC Collapse Experiments
}

\author{
E. A. Calzetta ${ }^{1}$ and B. L. $\mathrm{Hu}^{2 *}$ \\ ${ }^{1}$ Departamento de Fisica, FCEyN Universidad de Buenos Aires Ciudad Universitaria, 1428 Buenos Aires, Argentina \\ ${ }^{2}$ Department of Physics, University of Maryland, College Park, MD 20742, USA
}

(March 11, 2005)

\begin{abstract}
- Invited Talk presented at the Peyresq Meetings of Gravitation and Cosmology, 2003. To appear in Int. J. Theor. Phys.
\end{abstract}
\begin{abstract}
Main Theme We show that in the collapse of a Bose-Einstein condensate (BEC) ${ }^{1}$ certain processes involved and mechanisms at work share a common origin with corresponding quantum field processes in the early universe such as particle creation, structure formation and spinodal instability. Phenomena associated with the controlled BEC collapse observed in the experiment of Donley et al 2] (they call it 'Bose-Nova', see also [3]) such as the appearance of bursts and jets can be explained as a consequence of the squeezing and amplification of quantum fluctuations above the condensate by the dynamics of the condensate. Using the physical insight gained in depicting these cosmological processes, our analysis of the changing amplitude and particle contents of quantum excitations in these BEC dynamics provides excellent quantitative fits with the experimental data on the scaling behavior of the collapse time and the amount of particles emitted in the jets. Because of the coherence properties of BEC and the high degree of control and measurement precision in atomic and optical systems, we see great potential in the design of tabletop experiments for testing out general ideas and specific (quantum field) processes in the early universe, thus opening up the possibility for implementing 'laboratory cosmology'. ${ }^{2}$
\end{abstract}

Theoretical Cosmology in relation to General Relativity, Particle and Condensed Matter Physics For the last half a century the study of theoretical cosmology and high energy astrophysics has relied largely on general relativity and particle physics, while modern and contemporary cosmological exper-

\footnotetext{
*Emails: calzetta@df.uba.ar, hub@physics.umd.edu

${ }^{1}$ For an excellent introduction to BEC theory, see 1

${ }^{2}$ This essay has the same content as v2 of [4, with a few references updated. For more details, see [5].
}

iments are the fuse and the fuel of these activities. We witness the inception of nuclear astrophysics in the 50's, leading to the highly successful theories of neutron stars, and particle astrophysics in the 60's exemplified by the highly successful theory of nucleosynthesis which helped to establish the standard model in cosmology. Establishment of quantum field theory in curved spacetime [6] in the 70's laid the foundation for the study of quantum field processes in strong gravity, such as cosmological particle creation in the very early universe [7] and Hawking radiation in black holes [8] (Note these two processes contain very different physics). This pushed the frontiers of theoretical inquiries in leaps, back to the period after the Planck time. The inflationary cosmology [9] of the 80's also ushered in ideas of particle physics and quantum field theory, such as the decay of the false vacuum and the capability of vacuum energies driving the universe into ultrafast expansion, with scenarios radically different from the standard model.

This side of the story on the progress of modern cosmology with the help of gravitation theory and particle physics is well-known. What is perhaps lesser known or appreciated is the importance of ideas and techniques from condense matter physics and statistical mechanics in the study of cosmology of the early universe. We have seen the relevance of statistical mechanics, kinetic theory, stochastic processes and many-body dynamics in classical astronomy and physical cosmology (see, e.g., [10]). Here we want to emphasize the importance of ideas from condensed matter physics in conjunction with quantum field theory for treating early universe quantum processes, which is believed to have played a fundamental role in determining how spacetime and matter existing in different forms and states interplay, transform and evolve. The importance of 
viewing cosmology in the light of condensed matter physics, in terms of taking the correct viewpoints to ask the right questions, and approaches to understand the processes, has been called to our attention a long time ago (see, e.g., 11]). There were also proposals to study cosmological defect formation in helium experiments and to view cosmology as a critical phenomenon 12, 13. Similar efforts aim to identify analogs of full cosmological models 14. A recent monograph is devoted to the unity of forces at work in $\mathrm{He}^{3}$ droplets [15]. It should also be mentioned that the proposal of sonic black holes [16, 17] was perhaps the first analog model in black hole physics which stimulated recent activities in finding similar processes in fluids and condensed matter systems in the so-called analog gravity program [18].

Laboratory Cosmology Here we propose using the Bose-Einstein Condensate (BEC) and its dynamics as another useful venue to 'observe' and probe into some fundamental cosmological processes in the early universe. Specifically, we analyze the experiment performed by Donley et al. 2] on the controlled collapse of a BEC and identify the processes and mechanisms at work which are responsible for vacuum particle creation 7, structure formation 19] and spinodal instability (quenching) in phase transition 20 in the early universe. The collapsing BEC is the timereverse scenario of an expanding universe and the condensate plays a similar role as the vacuum in quantum field theory in curved spacetime. One can understand the production of atoms in the form of jets and bursts as the result of parametric amplification of vacuum fluctuations by the condensate dynamics. This is the same mechanism as cosmological particle creation from the vacuum, which is believed to be copious near the Planck time and during preheating after inflation 21]. Some basic ideas common to cosmological theories like "modes freeze when they grow outside of the horizon" can be used to explain the special behavior of jets and bursts ejected from the collapsing BEC. Finally the waiting time before a BEC starts to collapse obeys a scaling rule which can be derived from simple principles of spinodal instability in critical phenomena. These examples clearly indicate the great potential of a new field of research which we may call "laboratory cosmology", with tabletop experiments designed to test the workings of specific physical mechanisms in specific cosmological processes.
BEC Collapse Experiments In the experiment described by Donley et al. 2], a Bose-Einstein condensate (BEC) in a cold ( $3 \mathrm{nK})$ gas of Rubidium atoms is rendered unstable by a sudden inversion of the sign of the interaction between atoms. This is done by altering the binding energy at Feshbach resonance with an external magnetic field. After a waiting time $t_{\text {collapse, }}$ the condensate implodes, and a fraction of the condensate atoms are seen to oscillate within the magnetic trap which contains the gas. These atoms are said to belong to a 'burst'. After a time $\tau_{\text {evolve }}$ the interaction is suddenly turned off. For a certain range of values of $\tau_{\text {evolve }}$, new emissions of atoms from the condensate are observed. They are called 'jets'. Jets are distinct from bursts: they are colder, weaker, and have a characteristic disk-like shape. ${ }^{3}$

The Model The model is based on the Hamiltonian operator for $N$ interacting atoms with mass $M$ in a trap potential $V(\mathbf{r})=\left(\omega_{z}^{2} z^{2}+\omega_{\rho}^{2} \rho^{2}\right) / 2$, with radial $\rho$ and longitudinal $z$ coordinates measured in units 4 of $a_{h o}$, where $a_{h o}$ is a characteristic length of the trap, with associated (dimensionless) frequencies $\omega_{z}=$ $\omega_{\text {axial }} / \omega \sim 1 / 2$ and $\omega_{\rho}=\omega_{\text {radial }} / \omega \sim \sqrt{2}$. The interaction is assumed to be short ranged. We introduce a

\footnotetext{
${ }^{3}$ We call attention to the distinction between the 'Bose-Nova' 2 experiment studied here and other BEC collapse experiments 22 23. At magnetic fields around $160 \mathrm{G}$, where the effective scattering length is of the order of $500 a_{0}$ (and positive) $\left(a_{0}=\right.$ $0.52910^{-10} \mathrm{~m}$ is the Bohr radius) it is possible to observe oscillations between the usual atomic condensate and the molecular state [23] with a frequency of oscillations of hundreds of $\mathrm{KHz}$ [24]. By contrast, in the 'Bose-Nova' experiment [2] typical fields were around $167 \mathrm{G}$, the scattering length was only tens of Bohr radii (and negative) and the frequency of atom - molecule oscillations may be estimated as well over ten $\mathrm{MHz}$ 25. While coherent resonance between the atoms and the molecules is expected to exist for all of these experiments, and has been shown to play an important role in the outcomes of some 25, we deem it unlikely that it plays a dominant role in this experiment other than renormalizing the scattering length (For details, see [5]). Indeed no oscillations are reported in the original experimental paper. Instead, as this note shows, the primary mechanism for the Bose Nova phenomena is the parametric amplification of quantum fluctuations by the condensate dynamics, resulting in bursts and jets as particle production from (the squeezing of) the vacuum. Recent numerical simulations 26 and rigorous theoretical investigations [27] indicating the inadequacy of mean field theory seem to corroborate this view.

${ }^{4}$ We use a sign convention such that the effective coupling constant is positive for an attractive interaction, and a system of units where the length $a_{h o}$, time $t_{h o}$ and energy scale $E_{h o}=\hbar \omega=M \omega^{2} a_{h o}^{2}$ are defined with reference to the average frequency $\omega$. We work with units such that these three scales take the value 1 .
} 
dimensionless field operator $\Psi(r) \equiv a_{h o}^{-3 / 2} \Psi(x)$, and a dimensionless coupling constant $u=\left(\hbar \omega a_{h o}^{3}\right)^{-1} U=$ $4 \pi\left(a / a_{h o}\right)$.

$\Psi$ obeys the equation of motion $\dot{\Psi}=i[\hat{H}, \Psi]$ and satisfies the equal time commutation relations $\left[\Psi(t, \mathbf{r}), \Psi^{\dagger}\left(t, \mathbf{r}^{\prime}\right)\right]=\delta^{(3)}\left(\mathbf{r}-\mathbf{r}^{\prime}\right)$. We decompose the Heisenberg operator $\Psi=\Phi(\mathbf{r}, t)+\psi(\mathbf{r}, t)$ into a cnumber condensate amplitude $\Phi$ and a q-number noncondensate amplitude $\psi$, consisting of the fluctuations or excitations.

One crucial point of our analysis is that we shall focus on the evolution of the fluctuations for a given evolution of the condensate (as extracted from the experiments); in field theory terms, we shall work within the test field approximation. It is fair to say that a full theoretical account of the Bose - Nova experiment, describing the evolution of both condensate and fluctuations, does not exist. All we can say with any certainty is that we face here a strong back reaction regime, beyond the Hartree - Fock - Bogoliubov approximation [28.

In contradistinction, the dynamics of the fluctuations alone may be described, at least in the early stages of the experiment, by a simple Bogoliubov approximation 29. We obtain the equation of motion for the fluctuation field by subtracting from the full Heisenberg equation the Gross - Pitaievsky equation (GPE) for $\Phi$. We next parametrize the wave functions as $\Phi=\Phi_{0} e^{-i \Theta}, \psi=\psi_{0} e^{-i \Theta}$, where $\Phi_{0}$ and $\Theta$ are real. During the early stages of evolution, we may regard the condensate density as time independent, and the condensate phase as homogeneous, $\Phi_{0}=\Phi_{0}(r)$, $\Theta=\Theta(t)$. We may then write the equation for the fluctuation field

$$
\left[i \frac{\partial}{\partial t}-H+E_{0}\right] \psi_{0}+u \Phi_{0}^{2}\left(\psi_{0}+\psi_{0}^{\dagger}\right)=0
$$

where $E_{0}=\frac{1}{2}\left(\omega_{z}+2 \omega_{\rho}\right)$. As it is well known, this approximation is both "gapless" and "conserving" 30.

To solve equation $(\mathbb{1})^{5}$ we decompose $\psi_{0}$ into a selfadjoint and an anti-adjoint part $\psi_{0}=\xi+i \eta$, each part satisfying an equation

$$
\frac{\partial \xi}{\partial t}=\left[H-E_{0}\right] \eta
$$

${ }^{5}$ The squeezing of quantum unstable modes and its back reactions on the condensate has been considered before, e.g., as a damping mechanism for coherent condensate oscillations 31, and applied to the collapse of a homogeneous condensate in 32 .

$$
\frac{\partial \eta}{\partial t}+\left[H-E_{0}-2 u \Phi_{0}^{2}\right] \xi=0 .
$$

Since the trap Hamiltonian is time - independent, we have

$$
\frac{\partial^{2} \xi}{\partial t^{2}}+\left[H-E_{0}\right] H_{e f f} \xi=0 .
$$

Here $H_{\text {eff }}=H-E_{0}-2 u \Phi_{0}^{2}$. To have an unstable condensate it is necessary that at least one of the eigenvalues of $H_{e f f}$ is negative; the boundary of stability occurs when the lowest eigenvalue is exactly zero.

One further consideration is that we are interested in the part of the fluctuation field which remains orthogonal to the condensate. In the full theory, the condensate is the eigenfunction of the one - body density matrix with the largest (macroscopic) eigenvalue, and the non-condensate is built out of the other eigenstates 33. Since the one-body density matrix is Hermitian, they must be orthogonal. The ground state of $H_{\text {eff }}$ is certainly not orthogonal to the condensate, since neither have nodes. Observe that within our approximations, the noncondensate wave function is equivalent, up to a normalization, to the phonon operator in the particle- conserving formalism 34 .

If we adopt the values $\omega_{z}=1 / 2, \omega_{\rho}=\sqrt{2}$, relevant to the JILA experiment, then instability occurs when $\kappa=N_{0} a_{\text {crit }} / a_{\text {ho }}=0.51$. This result compares remarkably well with the experimental value $\kappa=0.55$ [2, 25], as well as with the theoretical estimate presented in Ref. [35. This agreement may be seen as natural, as the equations we postulate for the fluctuations may be obtained from the linearization of the GPE. In both calculations, the geometry of the trap plays a fundamental role.

Scaling of $t_{\text {collapse }}$ and Critical Dynamics As we have already noted, even for condensate densities above the stability limit, no particles are lost from the condensate during a waiting time $t_{\text {collapse }}$. Experimentally, $t_{\text {collapse }}$ is seen to get very large when the threshold of stability is approached from above, in a way which closely resembles the critical slowing down near the transition point characteristic of critical dynamics. In our problem, the quantity which plays the role of relaxation time is the characteristic time $\varepsilon^{-1}$ of exponential growth for the first unstable mode. This quantity diverges at the stability threshold, which in our analogy 
corresponds to the critical point. By dimensional analysis, we are led to the estimate $t_{\text {collapse }} \sim \varepsilon^{-1}$. Close to the critical point, we find

$$
t_{\text {collapse }}=t_{\text {crit }}\left(\frac{a}{a_{c r}}-1\right)^{-1 / 2}
$$

The power law Eq. (5) describes with great accuracy the way $t_{\text {collapse }}$ scales with the scattering length; the best fit to the experimental data is obtained for $t_{c r i t} \sim$ $5 \mathrm{~ms}$.

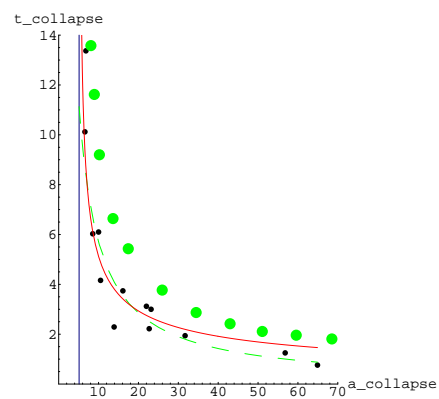

Figure 1:

In Fig. 1 we plot the scaling law (5) (full line) derived here and compare it with the experimental data for $N_{0}=6000$ as reported in Refs. 2] (small black points), the $t_{N L} \sim\left(u N_{0}\right)^{-1}$ prediction (suitably scaled) as given in 32 [36] (dashed line) and the results of numerical simulations reported in [37] (large grey dots). While all three theoretical predictions may be considered satisfactory, the $t_{N L} \sim\left(u N_{0}\right)^{-1}$ behavior fails to describe the divergence of $t_{\text {collapse }}$ as the critical point is approached, and the results of numerical simulations reported in 37] based on an improved Gross-Pitaevskii equation tend to be systematically above the experimental results, which may be a further indication of the quantum origin of this phenomenon 38 .

We wish to stress that our argument predicts the scaling exponent, but not the prefactor; even this apparently simple aspect of the Bosenova phenomenology is surprisingly resilient to theoretical explanation 28. The same scaling law is found from a different perspective in 39 .

Bursts and Jets as Amplified Quantum Fluctuations We now consider the evolution of quantum fluctuations, treated as a test field riding on the collapsing condensate whose dynamics is extracted from experiment. The initial state is defined by the condition that $u=0$ for $t<0$; we shall take it to be the particle vacuum $|0\rangle$, defined by $\psi_{0}(x, 0)|0\rangle=0$ everywhere.

One can introduce a mode decomposition of the $\xi$ operator based on the eigenfunctions of $\left[H-E_{0}\right] H_{\text {eff }}$. For short wavelengths $\lambda$, since $H \sim \lambda^{-2}>2 u \Phi_{0}^{2}$, we expect these eigenfunctions will approach the trap eigenmodes. The fact that particles in bursts are seen to oscillate with the trap frequencies [2] also suggests that their dynamics is determined by the trap Hamiltonian. Based on these observations we can assume a homogeneous condensate $2 u \Phi_{0}^{2} \sim \kappa^{-1} a \omega_{z} N_{0}(t)$, where $N_{0}(t)$ is the instantaneous total number of particles in the condensate. In practice, $\kappa^{-1}$ is a measure of the overlap between the condensate and the excitation modes. Therefore, the approximation may be improved by adjusting $\kappa$ according to the range of modes where it will be applied.

Let $\bar{N}_{0}$ be the initial number of particles in the condensate, and $a_{c r}=\kappa / \bar{N}_{0}$ the corresponding critical scattering length. Trap eigenfunctions $\psi_{\vec{n}}(r)$ are labeled by a string of quantum numbers $\vec{n}=\left(n_{z}, n_{x}, n_{y}\right)$. The eigenvalues of the trap Hamiltonian are (with the zero energy already subtracted) $E_{\vec{n}}=\omega_{z} n_{z}+$ $\omega_{\rho}\left(n_{x}+n_{y}\right)$. There are two kinds of modes, stable (oscillatory, or thawed) modes if $E_{\vec{n}}>\left(\frac{a}{a_{c r}}\right) \omega_{z}$, and unstable (growing, or frozen) modes if not. In the former case we find that, although we assume vacuum initial conditions, these modes do not remain empty. Up to $t_{\text {collapse, }}$, when the number of particles in the condensate is constant, the density

$$
\tilde{n}(r, t)=\frac{1}{8}\left(\frac{a}{a_{c r}}\right)^{2} \omega_{z}^{2} \sum_{\vec{n}} \psi_{\vec{n}}^{2}(r) \frac{\sin ^{2} \omega_{\vec{n}} t}{\omega_{\vec{n}}^{2}}
$$

(where $\omega_{\vec{n}}=\sqrt{E_{\vec{n}}\left[E_{\vec{n}}-\left(\frac{a}{a_{c r}}\right) \omega_{z}\right]}$ ) has a constant term and an oscillatory term. This oscillatory term is responsible for the appearance of 'bursts' of particles oscillating within the trap observed in the Bose-Nova experiment 2]. In the WKB limit it describes a swarm of particles moving along classical trajectories in the trap potential.

In the opposite case $E_{\vec{n}} \leq\left(\frac{a}{a_{c r}}\right) \omega_{z}$, the formulae for the density is obtained by the replacement of $\omega_{\vec{n}}$ in (6) by $i \sigma_{\vec{n}}$, thus $\omega_{\vec{n}}^{-1} \sin \omega_{\vec{n}} t \rightarrow \sigma_{\vec{n}}^{-1} \sinh \sigma_{\vec{n}} t$. Physically their difference is immense. In the first place, the 
density is growing exponentially, but unlike the previous case, there is no oscillatory component, and these particles do not oscillate in the trap, in the sense described above. These modes come alive at $\tau_{\text {evolve }}$ (as the scattering length is set to zero), whence they become ordinary trap modes which oscillate in the trap in the same way as the the burst modes. To the observer, they appear as a new ejection of particles from the core of the condensate, which makes up the socalled 'jets'. The sudden activation of a frozen mode (we are borrowing the language and concept of cosmological structure formation) by turning off the particle - particle interaction may be described as a "thaw".

Observe that in this picture several conspicuous features of jets become obvious. Jets may only appear if the turn - off time $\tau_{\text {evolve }}$ is earlier than the formation time of the remnant. Once the condensate becomes stable again, there are no more frozen modes to thaw. On the other hand, jets will appear (as observed) for $\tau_{\text {evolve }}<t_{\text {collapse }}$, when the condensate has not yet shed any particles. Also jets must be less energetic than bursts, since they are composed of lower modes.

Beyond $t_{\text {collapse }}$ the number of particles in the condensate, and therefore the instantaneous frequency of the excited modes, becomes time dependent. If we confine ourselves to the early stages of collapse we may assume nevertheless that the condensate remains homogeneous. Shifting the origin of time to $t_{\text {collapse }}$ for simplicity, we write $N_{0}(t)=\bar{N}_{0} \exp (-t / \tau)$ (see Fig. 2).

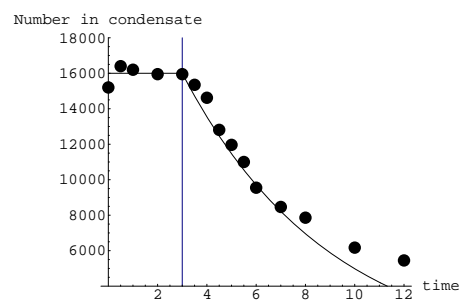

Figure 2:

After expanding in trap eigenmodes we find the two kinds of behavior described above. If $E_{\vec{n}}>\left(\frac{a \omega_{z}}{\bar{a}}\right)$, the mode is always oscillatory. If $E_{\vec{n}}<\left(\frac{a \omega_{z}}{\bar{a}}\right)$, the mode is frozen at $t_{\text {collapse }}$, but thaws when $\exp (-t / \tau) \sim$ $E_{\vec{n}} \bar{a} / a \omega_{z}$. During the frozen period, the modes are amplified, but they only contribute to bursts after thawing. If the evolution is interrupted while still frozen, they appear as a jet. We therefore conclude that the number of particles $N_{\text {jet }}$ in a jet at time $\tau_{\text {evolve }}$ is essen- tially the total number of particles in all frozen modes at that time. This is plotted in Fig 3 , for $\bar{N}_{0}=16,000$, $\omega_{\text {radial }}=110 \mathrm{~Hz}, \omega_{\text {axial }}=42.7 \mathrm{~Hz}, a=36 a_{0}$, and $\kappa=0.46$, and compared to the corresponding results as reported in 2 .

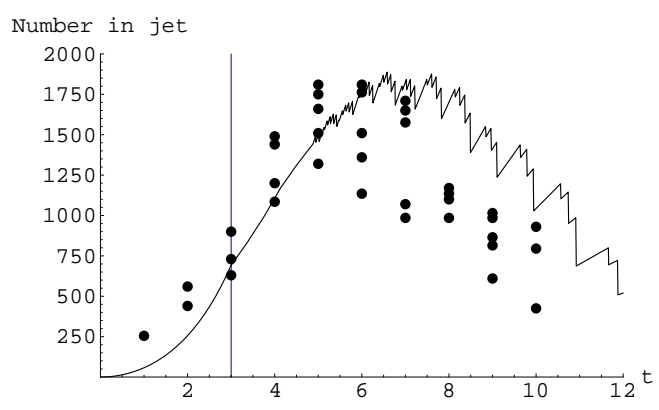

Figure 3:

We see that the agreement is excellent at early times (up to about $6 \mathrm{~ms}$ ). For later times, this model overestimates the jet population. This is due to the fact that, by not considering the shrinking of the condensate, we are overestimating the overlap between the condensate and the fluctuations, thus delaying the thaw. It nevertheless reproduces the overall slope of particle number with $\tau_{\text {evolve, }}$. It should also be remembered that we are computing the expected number of particles, but in the highly squeezed state which results from the frozen period, the fluctuations in particle number are comparable to the mean number itself.

It is interesting to observe that it is possible to reproduce the jets within a theory where the GPE equation is generalized to include an imaginary three - body recombination loss term 40. We do not regard this as necessarily a different explanation, but rather as a different way of handling the divide between condensate and non-condensate (the analogous cosmological problem would be whether to consider a spin two fluctuation generated during Inflation as a test field on the cosmological background, or as part of the geometry). Still agreement with the observed jets is obtained only for certain ranges of parameters, and it is unclear whether any single parameter set gives a satisfactory simultaneous account of all aspects of the experiment [28].

In this talk, we have presented a new viewpoint towards understanding the salient features in the physics of controlled collapse of a Bose-Einstein condensate described in the experiment of [2], i.e, in terms of quantum vacuum fluctuations parametrically amplified by 
the condensate dynamics. Even under a number of simplifying assumptions, our approach yields results in excellent agreement with experiment, particularly in the scaling of the waiting time $t_{\text {collapse }}$ and the number of particles in a jet. A background field separation is assumed here (even though the measured condensate dynamics contains the backreaction of noncondensates) so that one can treat these as test-field processes. A theoretical treatment of the fully self-consistent dynamics of both the condensate and its quantum fluctuations during collapse beyond the conventional Hartree - Fock - Bogolubov theory remains a worthy challenge (see, e.g., 41 and references therein).

If our explanation of the salient features of this experiment is correct one can think of using this process to create coherent atoms in highly squeezed states 42 . This is because the underlying mechanism of parametric amplification produces particles from vacuum fluctuations in squeezed states 43 .

Our way of thinking here is influenced by insights from the quantum field theory of particle creation and structure formation in cosmological spacetimes as well as theories of spinodal instability in phase transitions. One can conceivably design experiments with BEC dynamics to test out certain basic mechanisms and specific features of quantum processes in the early universe, thus opening a new venue for performing 'laboratory cosmology'.

Acknowledgement We thank Bill Phillips and Keith Burnett for kindling our interest in BEC dynamics, Elizabeth Donley and Jake Roberts for clarification of their data in the experiments described here and S. Kokkelmans for communicating key unpublished data (at the time the letter 4 on which this essay is based was written). We thank our colleagues Ted Jacobson, Stefano Liberati at Maryland and Charles Clark and his group members at NIST Gaithersburg during our seminars for their questions and comments. EC acknowledges discussions with Eric Bolda. This research is supported in part by NSF grant PHY03-00710 and by CONICET, UBA, Fundacion Antorchas and ANPCyT under grant PICT99 03-05229.

\section{References}

[1] C. Pethick and H. Smith, Bose-Einstein condensation in dilute gases (Cambridge University Press, Cambridge, England, 2002).
[2] E. Donley et. al., Nature 412, 295 (2001); N. Claussen, Ph. D. Thesis, U. of Colorado (2003); N. Claussen et al., Phys. Rev. A67, 060701(R) (2003).

[3] J. Chin, J. Vogels and W. Ketterle, Phys. Rev. Lett. 90, 160405 (2003).

[4] E. Calzetta and B. L. Hu, "Bose-Novae as Squeezing of the Vacuum by Condensate Dynamics" cond-mat/0208569

[5] E. Calzetta and B. L. Hu, Phys. Rev. A68 (2003) 043625 cond-mat/0207289

[6] N. D. Birrell and P. C. W. Davies, Quantum fields in curved spaces (Cambridge University Press, Cambridge, England, 1982).

[7] J. Schwinger, Phys. Rev. 82, 664 (1951); L. Parker, Phys. Rev. 183, 1057 (1969); Ya. Zel'dovich, Pisma Zh. Eksp. Teor. Fiz 12, 443 (1970) (Eng. trans. Sov. Phys.- JETP Lett. 12, 307 (1971)).

[8] S. W. Hawking, Nature 248, 30 (1974); Com. Math. Phys. 43, 199 (1975).

[9] A. H. Guth, Phys. Rev. D23, 347 (1981).

[10] J. P. E. Peebles, Principles of Physical Cosmology (Princeton University Press, 1993)

[11] B. L. Hu, "Cosmology as 'Condensed Matter' Physics" in Proc. Third Asia Pacific Physics Conference, ed. Y. W. Chan et al (World Scientific, Singapore, 1988) Vol. 1, p. 301. gr-qc/9511076.

[12] W. Zurek, Phys. Rep. 276, 178 (1996).

[13] L. Smolin, "Cosmology as a Problem in Critical Phenomena" in Complex Systems and Binary Networks eds. L. Lopez-Pena et al (Springer, Berlin, 1995) gr-qc/9505022

[14] P. Fedichev and U. Fischer, Phys. Rev. A69, 033602 (2004); U. Fischer and R. Schützhold, cond-mat/0406470.

[15] G. Volovik, Universe in a Helium Droplet (Oxford University Press, Oxford, 2003).

[16] W. G. Unruh, Phys. Rev. Lett. 46, 1351 (1981); Phys. Rev. D51, 2827 (1995)

[17] T. Jacobson, Phys. Rev. D44, 1731 (1991); Phys. Rev. D53, 7082 (1994) 
[18] L. Garay, J. Anglin, J. Cirac, and P. Zoller, Phys. Rev. Lett. 85, $4643-4647$ (2000); C. Barcelo, S. Liberati and M. Visser, Phys. Rev. A68, 053613 (2003); Class. Quant. Grav. 18 (2001) 1137; T. Jacobson and T. Koike, in "Artificial Black Holes", eds. M. Novello, M. Visser, and G. Volovik (World Scientific, 2002) (cond-mat/0205174); R. Schtzhold and W. G. Unruh, Phys. Rev. D 66, 044019 (2002).

[19] E.g., J. Bardeen, Phys. Rev. D22, 1882 (1980); V. Mukhanov, H. Feldman and R. Brandenberger, Phys. Rep. 215, 203 (1992) and references therein.

[20] E. Calzetta, Ann. Phys. (N.Y.) 190, 32 (1989); F. Cooper, Y. Kluger, E. Mottola and J. P. Paz, Phys. Rev. D51, 2377 (1995).

[21] L. Kofman, A. Linde, A. Starobinsky, Phys. Rev. Lett. 73 (1994) 3195; Phys. Rev. D56, 3258 (1997) and referencs therein. Y. Shtanov, J. Traschen and R. Brandenberger, Phys. Rev. D 51, 5438 (1995). H. Fujisaki et al., Phys. Rev. D53, 6805 (1996). S. A. Ramsey and B. L. Hu, Phys. Rev. D56, 678 (1997); D. Chung et al., Phys. Rev. D62, 043508 (2000) T. Charters, A. Nunes and J. Mimoso, hep-ph/0502053

[22] J. Roberts et al., Phys. Rev. Lett. 81, 5109 (1998); S. Cornish et al., Phys. Rev. Lett. 85, 1795 (2000).

[23] N. Claussen et al., Phys. Rev. Lett. 89, 10401 (2002); E. Donley et al., Nature 417, 529 (2002).

[24] S. Kokkelmans and M. Holland, Phys. Rev. Lett. 89, 180401 (2002); M. Mackie, K. Suominen and J. Javainen, Phys. Rev. Lett. 89, 180403 (2002); cond-mat/0209083 M. Mackie et al., physics/0210131

[25] N. Claussen et al., cond-mat/0302195 S. Kokkelmans, private communication.

[26] C. Savage, N. Robins and J. Hope, cond-mat/0207308

[27] R. Duine and H. Stoof, Phys. Rev. A68, 013602 (2003).

[28] S. Wüster, J. Hope and C. Savage, cond-mat/0412041

[29] J. Andersen, Rev. Mod. Phys. 76, 599 (2004).
[30] P. Hohenberg and P. Martin, Ann Phys 34, 291 (65); A. Griffin, Phys. Rev. B 53, 9341 (96); H. Shi and A. Griffin, Phys. Rep. 304, 1 (1998); Yu. Ivanov, F. Riek and J. Knoll, hep-ph/0502146.

[31] Yu. Kagan and L. Maksimov, Phys. Rev. A64, 53610 (2001).

[32] V. A. Yurovsky, Phys. Rev. A65, 33605 (2002). V. A. Yurovsky A. Ben-Reuven, cond-mat/0205267

[33] O. Penrose and L. Onsager, Phys. Rev. 104, 576 (1956).

[34] M. Girardeau and R. Arnowitt, Phys. Rev. 113, 755 (1959); Y. Castin and R. Dum, Phys. Rev. Lett. 79, 3553 (1997); Y. Castin and R. Dum, Phys. Rev. A57, 3008 (1998); C. Gardiner, Phys. Rev. A56, 1414 (1997); S. Gardiner, D. Jaksch, R. Dum, J. Cirac and P. Zoller, Phys. Rev. A62, 23612 (2000); S. Morgan, cond-mat/0307246 Z. Idziaszek, cond-mat/0406052.

[35] A. Gammal, T. Frederico and L. Tomio, Phys. Rev. A64, 55602 (2001).

[36] M. Trippenbach, Y. Band and P. Julienne, Phys. Rev. A62, 23608 (2000).

[37] H. Saito and M. Ueda, cond-mat/0305242

[38] G. Berman, A. Smerzi and A. Bishop, Phys. Rev. Lett. 88, 120402 (2002).

[39] S. Métens, G. Dewel and P. Borckmans, Phys. Rev. A68, 045601 (2003).

[40] L. Santos and G. Shlyapnikov, Phys. Rev. A66, 011602 (R) (2002); W. Bao, D. Jaksch and P. Markovich, J. Phys. B At. Mol. Opt. Phys. 37, 329 (2004); SK Adhikari, J. Phys. B At. Mol. Opt. Phys. 37, 1185 (2004).

[41] A. M. Rey et al., Phys. Rev. A69, 033610 (2004).

[42] J. Rogel-Salazar et al., cond-mat/0110076

[43] L. Grischuk and Y. V. Sidorov, Phys. Rev. D42, 3413 (1990). B. L. Hu, G. Kang and A. Matacz, Int. J. Mod. Phys. A9, 991 (1994). 